\title{
Antibiotic-producing ability by representatives of a newly discovered lineage of actinomycetes
}

\author{
Elena Busti, Paolo Monciardini, Linda Cavaletti, Ruggiero Bamonte, \\ Ameriga Lazzarini, Margherita Sosio and Stefano Donadio†
}

Correspondence

Stefano Donadio

stefano_donadio@libero.it

Received 11 July 2005

Revised 9 November 2005

Accepted 11 December 2005

\author{
Vicuron Pharmaceuticals, via R. Lepetit 34, 21040 Gerenzano, Italy
}

\begin{abstract}
The discovery of new antibiotics and other bioactive microbial metabolites continues to be an important objective in new drug research. Since extensive screening has led to the discovery of thousands of bioactive microbial molecules, new approaches must be taken in order to reduce the probability of rediscovering known compounds. The authors have recently isolated slow-growing acidophiles belonging to the novel genera Catenulispora and Actinospica within the order Actinomycetales. These strains, which likely belong to a new suborder, grow as filamentous mycelia, have a genome size around $8 \mathrm{Mb}$, and produce antimicrobial activities. In addition, a single strain harbours simultaneously genes encoding type I and type II polyeketide synthases, as well as non-ribosomal peptide synthetases. The metabolite produced by one strain was identified as a previously reported dimeric isochromanequinone. In addition, at least the Catenulispora strains appear globally distributed, since a PCR-specific signal could be detected in a significant fraction of acidic soils from different continents, and similar strains have been independently isolated from an Australian soil (Jospeh et al., App/ Environ Microbio/ 69, 7210-7215, 2003). Thus, these previously uncultured actinomycetes share several features with Streptomyces and related antibiotic-producing genera, and represent a promising source of novel antibiotics.
\end{abstract}

\section{INTRODUCTION}

Programmes aimed at the discovery of antibiotics and other bioactive metabolites from microbial sources have yielded an impressive number of compounds over the past 50 years, many of which have found applications in human medicine and agriculture. However, it soon became apparent during screening programmes that some microbial metabolites were discovered more frequently than others. As the number of described microbial metabolites increased, so did the probability of rediscovering known compounds. At the present time, with several thousands of described microbial metabolites, strategies must be introduced into screening programmes to increase the chances of discovering novel compounds (Bull et al., 2000; Lancini et al., 1995). The filamentous actinomycetes account for a significant fraction of microbial metabolites and, among them, Streptomyces is by far the most prolific genus. The pharmaceutical industry, over several decades, has probably isolated and screened millions of Streptomyces strains. Consequently, the chances of isolating a novel Streptomyces strain have substantially diminished, and so has the probability of discovering a novel

tPresent address: KtedoGen, via Cav. Brusa 43, 21046 Malnate, Italy.

Abbreviations: ITS, internal transcribed spacer; NRPS, non-ribosomal peptide synthetase; PKS, polyketide synthase.

The GenBank/EMBL/DDBJ accession numbers for the sequences reported in this paper are given in Tables 1-3. compound. Therefore, while it has been estimated that only a fraction of the antibiotics produced by Streptomyces strains have been discovered (Watve et al., 2001), identifying the undiscovered portion will require a substantial effort.

Genomic studies indicate that the genetic potential for producing secondary metabolites is not uniformly distributed within the bacterial world. In fact, most bacterial genomes lack any detectable gene cluster for secondary metabolism. On the other hand, Streptomyces coelicolor (Bentley et al., 2002) and Streptomyces avermitilis (Ikeda et al., 2003; Omura et al., 2001) each possess more than 20 gene clusters devoted to the synthesis of secondary metabolites. Multiple gene clusters for secondary metabolism appear also to be a hallmark of several non-Streptomyces filamentous actinomycetes (Sosio et al., 2000) and of the myxobacteria (Gerth et al., 2003). It is interesting to point out that genomic data parallel the observed discovery events and indicate that streptomycetes, pseudomonads and bacilli have yielded large numbers of metabolites since they can be easily isolated and have a relatively large genetic potential for producing secondary metabolites. It is thus likely that most microbial strains that could be isolated in large numbers have actually been screened for bioactivities.

Historically, significant advances in the discovery of new antibiotics occurred when previously little-known actinomycete genera were isolated in high numbers and rapidly 
screened (Parenti \& Coronelli, 1979; Wagman \& Weinstein, 1980). In recent years, the family Micromonosporaceae has been enlarged by the isolation of previously unreported genera from marine sources (Magarvey et al., 2004; Mincer et al., 2002). We also reasoned that previously uncultured actinomycete strains might represent a potential source of novel bioactive metabolites (Donadio et al., 2005), and have recently proposed the new genera Catenulispora (Busti et al., 2006) and Actinospica (Cavaletti et al., 2006), which belong to the novel suborder Catenulisporinae (Cavaletti et al., 2006). However, frequent production of secondary metabolites is confined to selected families within the order Actinomycetales. Here, we show that representatives of the genera Catenulispora and Actinospica share several features typical of antibiotic-producing Streptomyces and related actinomycetes.

\section{METHODS}

Soils, strains and culture conditions. All soil samples were from the historical collection in our laboratories. Samples were air-dried at $30^{\circ} \mathrm{C}$ for $2-7$ days and stored in a sealed plastic container before use. Source 11943 is an acidic ( $\mathrm{pH} 4 \cdot 2)$ soil sample collected from a temperate forest in Gerenzano, Italy. Source 8505 is an acidic soil sample ( $\mathrm{pH} \mathrm{5 \cdot 0)}$ ) from Nicaragua. Source 12228 ( $\mathrm{pH} \mathrm{6 \cdot 8)}$ ) was collected in a chestnut wood near Salerno, Italy.

The strains used in this study are reported in Table 1 . They were isolated in three distinct campaigns from independent aliquots of soils 11943 and 8505. Strains were maintained on HSA (Suzuki et al., 2001), BTT-agar or ISP3 plates (Atlas \& Park, 1993), all adjusted to $\mathrm{pH} 4 \cdot 5-5 \cdot 8$ with $\mathrm{HCl}$ after autoclaving. As liquid media, BTT and ATSB (Busti et al., 2006) were used for the Neo and Gamma strains, respectively. Both media were adjusted to $\mathrm{pH} 6$ with $\mathrm{HCl}$ before autoclaving. Growth temperature was $28^{\circ} \mathrm{C}$ and, depending on inoculum, strains required 4-21 days for vigorous growth.

Phylogenetic and automated ribosomal intergenic spacer analyses. Genomic DNA was extracted with the GenElute Bacterial Genomic DNA kit (Sigma). PCR amplification and sequence of the $16 \mathrm{~S}$ rRNA gene, and phylogenetic analyses, were performed as described by Monciardini et al. (2002). The 16S-23S internal transcribed spacer (ITS) was amplified as described by Mazza et al. (2003), except that the forward primer was $5^{\prime}$-end labelled with the fluorochromes NED (yellow), FAM (blue) and HEX (green). The sizes of amplified PCR products were determined by capillary electrophoresis using an ABI 310 genetic analyser (Applied Biosystems). Sample preparation and run conditions were as described by Fisher \& Triplett (1999). The internal size standard was GeneScan-1000 and data were analysed by using the GENESCAN 3.1 software (Applied Biosystems). Only the number and apparent sizes of peaks were considered; relative peak area was not used for strain comparison. Sizes obtained in different runs for the three independent PCR products corresponding to each strain (each labelled with a different dye) were compared, and peak size was calculated as the mean of the three measures.

Genome size determination. Strains were grown under shaking conditions until sufficient growth was observed. At this point, $3 \mathrm{~mm}$ glass beads were added, and flasks were shaken for a further 3-7 h. Then the dispersed mycelium was reinoculated into fresh medium and grown for a further 1-3 days. Agarose plugs were prepared by a modification of the procedure described by Beyazova et al. (1995). Briefly, mycelia were harvested by centrifugation and, if necessary,
Table 1. Strains analysed

\begin{tabular}{|lrll|}
\hline Strain & Source $^{\star}$ & $\begin{array}{c}\text { 16S rRNA } \\
\text { accession no. }\end{array}$ & \multicolumn{1}{c|}{ ITS size(s) $\dagger$} \\
\hline Neo1 & 11943 & AJ865855 & 565 \\
Neo2 & 11943 & AJ865856 $\ddagger$ & 565,579 and 582 \\
Neo3\$ & 11943 & AJ865857II & 566 and 582 \\
Neo6 & 11943 & AJ865858 & 561 and 567 \\
Neo15 & 11943 & AJ865860 $\ddagger$ & 565 and 575 \\
Neo9 & 11943 & AJ865859II & 565 and 579 \\
Gamma3 & 11943 & AJ865861 & 637 \\
Gamma4 & 11943 & AJ865862 & 637 and 641 \\
Gamma6 & 11943 & AJ865863\# & 574 and 619 \\
Gamma25 & 8505 & AJ865864 & 590,592 and 623 \\
Gamma22 & 11943 & AM157335\# & 574 and 619 \\
Gamma26 & 8505 & AJ865865 & $587,593,621$ and 626 \\
Gamma28 & 8505 & AJ865866\# & 589 and 623 \\
\hline
\end{tabular}

${ }^{\star}$ Numbers correspond to the internal source collection codes.

$\dagger$ Mean size (nt) of the ITS peak(s), rounded to the nearest unit. $\ddagger$ Strains Neo2 and Neo15 have identical 16S rRNA gene sequences. $\S$ Strain Neo3 corresponds to C. acidiphila ID139908 ${ }^{\mathrm{T}}$ (Busti et al., 2006).

IIStrains Neo3 and Neo9 have identical 16S rRNA gene sequences. Strains Gamma3 and Gamma6 correspond to A. acidiphila GE $134766^{\mathrm{T}}$ and $A$. robiniae $\mathrm{GE} 134769^{\mathrm{T}}$, respectively (Cavaletti et al., 2006).

\#Strains Gamma6 and Gamma22 have identical 16S rRNA gene sequences.

frozen at $-80{ }^{\circ} \mathrm{C}$ in $10 \%(\mathrm{v} / \mathrm{v})$ glycerol. After thawing, cells were washed twice with $0 \cdot 5 \mathrm{M}$ EDTA pH 8 and resuspended in $10 \mathrm{ml}$ of the same buffer. After addition of $2 \mathrm{ml}$ phenol saturated with TE (10 $\mathrm{mM}$ Tris/HCl, $1 \mathrm{mM}$ EDTA, $\mathrm{pH} 8)$ and a $15 \mathrm{~min}$ incubation at $65^{\circ} \mathrm{C}$, cells were washed three times with chloroform/isoamyl alcohol $(24: 1, \mathrm{v} / \mathrm{v})$ and then incubated in $2 \%$ SDS, $2 \mathrm{mM}$ EDTA for $15 \mathrm{~min}$ at $65^{\circ} \mathrm{C}$. After washing once with $1 \mathrm{mg} \mathrm{BSA} \mathrm{ml}^{-1}$ in $5 \mathrm{mM}$ EDTA and three times with TE, the harvested cells were suspended in TEsuc $\left(0.3 \mathrm{M}\right.$ sucrose in TE) to obtain a final $\mathrm{OD}_{600}$ between 2 and 3. The cell suspension was mixed with an equal volume of melted $2 \%$ low-melting-point agarose and kept at $50{ }^{\circ} \mathrm{C}$ before pouring into plug moulds, where it was allowed to solidify at $4{ }^{\circ} \mathrm{C}$. Agarose plugs were treated with $1 \mathrm{mg}$ lysozyme $\mathrm{ml}^{-1}$ in TEsuc for $2-3 \mathrm{~h}$ at $37^{\circ} \mathrm{C}$, then overnight with $1 \mathrm{mg}$ subtilisin $\mathrm{ml}^{-1}$ in $\mathrm{TE}$ at $50{ }^{\circ} \mathrm{C}$. Finally, plugs were washed three times, $1 \mathrm{~h}$ each, with TE at $50{ }^{\circ} \mathrm{C}$. Restriction digestions were performed by pre-incubating plugs in $500 \mu \mathrm{l}$ of suitable buffer for $15 \mathrm{~min}$ at $37^{\circ} \mathrm{C}$, followed by $5-6 \mathrm{~h}$ at $37^{\circ} \mathrm{C}$ with $40 \mathrm{U}$ of enzyme with occasional shaking (for Asel, after 2-3 $\mathrm{h}$ an additional $10 \mathrm{U}$ were added).

Pulsed-field gel electrophoresis was performed on $1 \%$ agarose gels (PFGE certified, Bio-Rad) in $0.5 \times$ TBE (Tris/borate/EDTA; Sambrook \& Russell, 2001), containing $50 \mathrm{mg}$ thiourea $1^{-1}$, in a CHEF-DRIII apparatus (Bio-Rad). Running conditions were $6 \mathrm{~V} \mathrm{~cm}^{-1}$ at $14^{\circ} \mathrm{C}$. The run consisted of $15 \mathrm{~h}$ at a switch time of $70 \mathrm{~s}$ and a field angle of $120^{\circ}$, followed by $11 \mathrm{~h}$ at a switch time of $120 \mathrm{~s}$ and a field angle of $120^{\circ}$. Fragment sizes were estimated with the help of size standards such as Saccharomyces cerevisiae chromosomes (BioLabs) or AseI-digested Streptomyces coelicolor genomic DNA, prepared as described by Kieser et al. (1992). 
Amplification of secondary metabolism genes. For the amplification of the $\sim 700 \mathrm{bp}$ segment encoding the KS domain from modular polyketide synthases (PKS-I), we used the primers described by Courtois et al. (2003), with a slight modification of their PCR protocol. Briefly, the PCR mixture consisted of $500 \mathrm{nM}$ each primer, $200 \mu \mathrm{M}$ dNTPs, $10 \mathrm{mM}$ Tris/HCl $(\mathrm{pH} 8.3), 2.5 \mathrm{mM}$ $\mathrm{MgCl}_{2}, 50 \mathrm{mM} \mathrm{KCl}, 7 \%$ dimethyl sulfoxide, $2 \mathrm{U}$ RedTaq DNA polymerase and 10-20 ng genomic DNA, in a final volume of $50 \mu \mathrm{l}$. After a $5 \mathrm{~min}$ incubation at $95^{\circ} \mathrm{C}$, three cycles (each consisting of $45 \mathrm{~s}$ at $95^{\circ} \mathrm{C}, 45 \mathrm{~s}$ at $65^{\circ} \mathrm{C}$ and $1 \mathrm{~min}$ at $72^{\circ} \mathrm{C}$ ) were followed by 30 cycles under identical conditions, except that the annealing temperature was lowered to $62^{\circ} \mathrm{C}$. To amplify a portion of the $\sim 600 \mathrm{bp}$ segment encoding KS $\alpha$ from PKS-II genes, we used the conditions described by Metsä-Ketelä et al. (1999). To amplify the 450 bp segment encoding the adenylation (A) domain of non-ribosomal peptide synthetases (NRPSs), we used the primers ADEdom5 ( $5^{\prime}$ ACSGGCNNNCCSAAGGGCGT-3') and ADEdom3 (5'-CTCSGTSGGSCCGTA- $\left.3^{\prime}\right)$. The PCR mixture was as above, except for $1 \mu \mathrm{M}$ each primer, $1.5 \mathrm{mM} \mathrm{MgCl}_{2}$ and $3 \% \mathrm{DMSO}$. After a 5 min incubation at $96^{\circ} \mathrm{C}, 10$ cycles (each of $1 \mathrm{~min}$ at $95^{\circ} \mathrm{C}, 45 \mathrm{~s}$ at $50^{\circ} \mathrm{C}$ and $1 \mathrm{~min}$ at $72{ }^{\circ} \mathrm{C}$ ) were followed by 20 cycles under identical conditions, except that the annealing temperature was raised to $55^{\circ} \mathrm{C}$. All PCR reactions were terminated with a 10 min elongation at $73^{\circ} \mathrm{C}$.

PCR products were cloned in the pGEMT-Easy vector (Promega), and the ligation mixture was used to transform E. coli by standard procedures (Sambrook \& Russell, 2001). Plasmids were recovered with the Genelute Plasmid Miniprep Kit (Sigma) and inserts sequenced using vector primers. Sequences were compared to GenBank through FASTA (Pearson, 1990). All other sequence analyses were performed with programs of the Wisconsin Package, Version 9.1 (Genetics Computer Group). Phylogenetic analyses were performed with programs of the PHYLIP package (Felsenstein, 1993). Putative substrate specificity of A domains was determined by comparison with the NRPS8AA database maintained at the NP Biogene web site (http:// www.npbiogene.com).

Antimicrobial activities. The production of bioactive metabolites was assayed against Bacillus subtilis (ATCC 6633), Staphylococcus aureus (L819), Micrococcus luteus (ATCC 9341) and Escherichia coli (L47) on AM1 plates containing $5 \times 10^{5}$ c.f.u. $\mathrm{ml}^{-1}$ of the test micro-organism. All strains were from the Vicuron strain collection. Bioactivity was determined employing either agar plugs cut from 7-10-day-old plates, or $10 \mu \mathrm{l}$ from a 4 -day broth culture.

For metabolite extraction, strains Neo3 and Gamma22 were grown on a BTT or ISP3 agar plate, respectively, for $7-10$ days at $28^{\circ} \mathrm{C}$. Then $7 \mathrm{ml}$ methanol per g solid medium was added and thoroughly mixed; after agar removal, the resulting liquid phase was extracted twice with 1 vol. ethyl acetate. The organic phase was dried under vacuum and dissolved in $0.5 \mathrm{ml} 10 \%$ DMSO. An aliquot was fractionated by HPLC using a $5 \mu$-symmetry $\mathrm{C}_{18}$ column (Waters Chromatography), and the effluent was subjected to mass spectrometry and diode-array UV-VIS analysis. Fractions from the HPLC column were collected and tested for biological activity against Staph. aureus. The UV and MS parameters of the biologically active fraction(s) were searched against Vicuron's proprietary database of microbial products (Lazzarini et al., 2000).

Neo-specific PCR protocol. Following described procedures (Monciardini et al., 2002), the 16S rRNA gene sequences from $\mathrm{Neo}$ strains (Table 1 and additional isolates) were used to identify the group-specific primers NeoF178 (5'-GGGTGGAAAGTTTTTCGGTGGA-3') and NeoR940 (5'-CCCCACATCTCTGCAGGTAG-3'), which are expected to yield a 762 bp PCR product. The PCR mixture consisted of $500 \mathrm{nM}$ each primer, $200 \mu \mathrm{M}$ each dNTP, $10 \mathrm{mM}$ Tris/HCl (pH 8.3), $1.5 \mathrm{mM} \quad \mathrm{MgCl}_{2}, 50 \mathrm{mM} \mathrm{KCl}, 3 \mu \mathrm{l} 10 \times$ Denhardt's solution, 2 U RedTaq DNA polymerase and 5 ng DNA, in a final volume of $50 \mu \mathrm{l}$. Cycling parameters were as follows: 5 min denaturation at $95^{\circ} \mathrm{C}$, followed by 30 cycles of $45 \mathrm{~s}$ at $94^{\circ} \mathrm{C}$, $30 \mathrm{~s}$ at $65^{\circ} \mathrm{C}$ and $1 \mathrm{~min}$ at $72^{\circ} \mathrm{C}$, followed by a final extension of $10 \mathrm{~min}$ at $72{ }^{\circ} \mathrm{C}$. For amplification directly from soil DNA, conditions were as above, except that 16 cycles, each consisting of $45 \mathrm{~s}$ at $94{ }^{\circ} \mathrm{C}, 30 \mathrm{~s}$ at $65^{\circ} \mathrm{C}$ and $1 \mathrm{~min}$ at $72^{\circ} \mathrm{C}$ (but decreasing the annealing temperature by $0.25^{\circ} \mathrm{C}$ after each cycle), were followed by 20 cycles, each consisting of $30 \mathrm{~s}$ at $94^{\circ} \mathrm{C}, 30 \mathrm{~s}$ at $60 \cdot 5^{\circ} \mathrm{C}$ and $1 \mathrm{~min}$ at $72{ }^{\circ} \mathrm{C}$. The PCR protocol, validated as described by Monciardini et al. (2002) using genomic DNA from several actinomycete strains, showed a sensitivity of 1-5 pg, estimated with purified DNA from Neo strains. The selectivity of the PCR protocol was assessed on soil DNA, by end-sequencing 17 independent clones obtained after cloning the PCR product from sources 11943 and 12228. All sequenced clones showed between $99 \cdot 1$ and $100 \%$ identity to those of the Neo strains from Table 1 (data not shown).

Nucleotide accession numbers. The sequences described here have been deposited in GenBank, and the corresponding accession numbers are reported in Tables 1-3.

\section{RESULTS}

\section{Strain diversity}

Descriptions of Catenulispora acidiphila and of Actinospica robiniae and A. acidiphila are reported elsewhere (Busti et al., 2006; Cavaletti et al., 2006). The strains analysed in this study were derived from three independent isolation campaigns from two different sources and are referred to with the Neo- and Gamma- prefixes to designate Catenulisporaand Actinospica-related strains, respectively (Table 1). All strains show the typical characteristics of filamentous actinomycetes, with branching vegetative hyphae and production of aerial hyphae that differentiate into spore chains upon ageing.

We analysed the isolates in order to assess the level of phylogenetic diversity. The strains were initially grouped according to their ITSs, applying to single strains the technique originally developed for community analysis (Fisher \& Triplett, 1999). For each strain, the ITS was PCRamplified and the apparent sizes of the amplified products were recorded. We analysed 29 Gamma strains (isolated from sources 8505 and 11943) and 35 Neo strains (isolated from source 11943 only). Each strain was characterized by a reproducible pattern, comprising one to four peaks of different sizes (data not shown). For each lineage, six distinct ITS profiles were obtained. Next, we randomly chose one representative strain from each profile type, leading to the 12 strains with unique profiles reported in Table 1, which were further characterized through $16 \mathrm{~S}$ rRNA sequence analysis (Fig 1).

For the six Neo strains, 16S rRNA variability was low, with pairwise sequence identity between 99.6 and $100 \%$. The Australian isolates Ellin5034, -5119 and -5116 (Joseph et al., 2003 ) have $16 \mathrm{~S}$ rRNAs highly similar (99.3-99.8\% pairwise identity) to those of the six Neo strains. The Gamma strains were divided into two groups: one consisting of Gamma3 and -4 (sharing $99 \cdot 6 \%$ sequence identity), and the second 


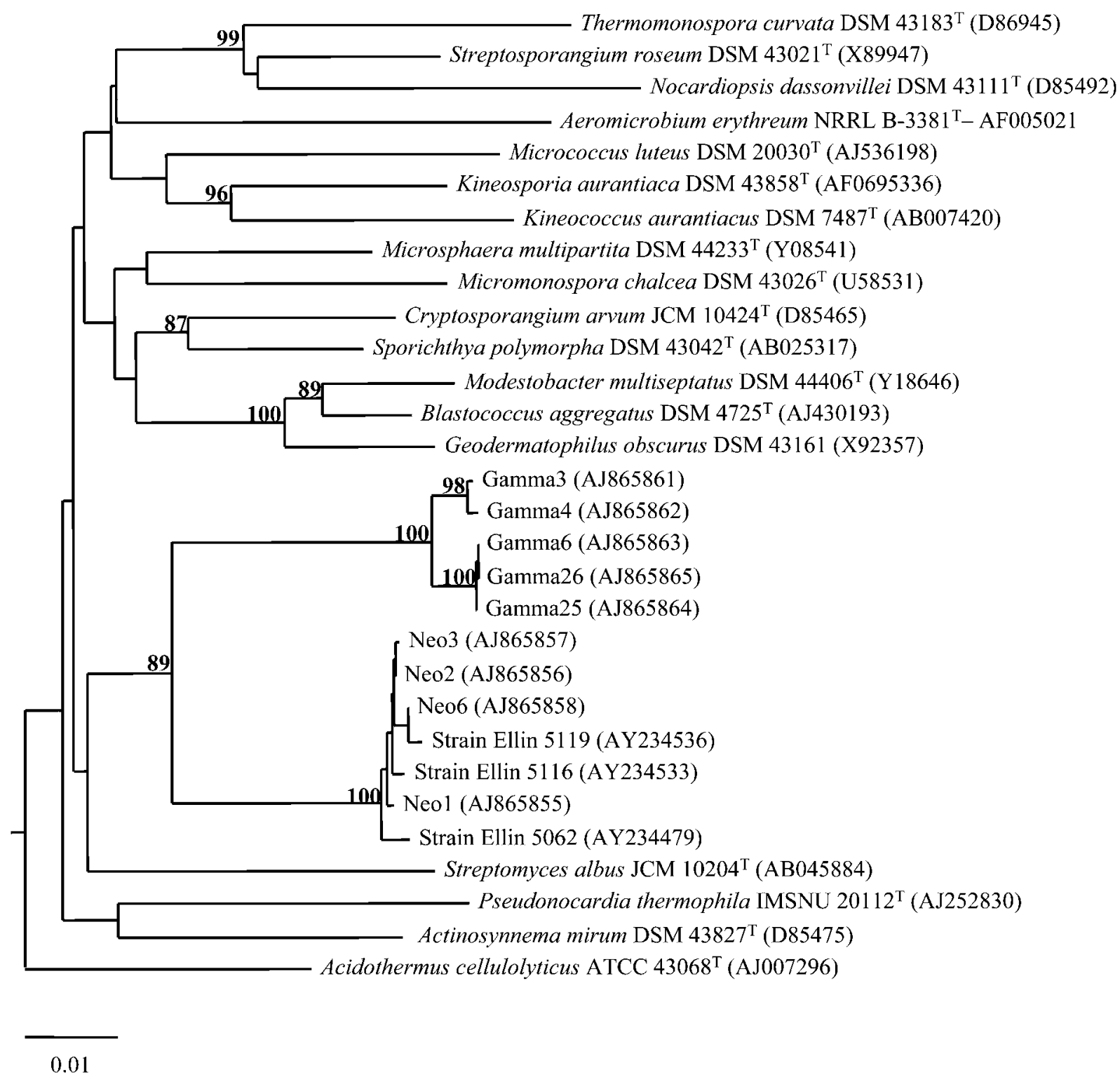

Fig. 1. Phylogenetic tree of $16 \mathrm{~S}$ rRNA genes from Catenulispora and Actinospica strains, rooted using the $16 \mathrm{~S}$ rRNA sequence of Conexibacter woesei DSM $14684^{\top}$ (GenBank AJ440237). The tree was constructed substantially as described by Cavaletti et al. (2006). Accession numbers for the sequences are in parentheses. Numbers at nodes are bootstrap values based on 100 resamplings; values lower than 70 are not shown. The scale bar indicates 1 inferred nucleotide substitution per $100 \mathrm{nt}$.

consisting of the other four strains (99-8-100\% identity). It should be noted that Gamma 6 and -28 , characterized by $99.9 \%$ identical $16 \mathrm{~S}$ sequences, were isolated from soils collected from locations far apart (Table 1).

\section{Genome size}

Agarose-embedded DNA from Neo1 and Neo2 was prepared as described in Methods, and restricted with AseI, DraI and SspI. Neo1 and Neo2 DNAs yielded distinct fragments when cut with AseI and DraI, while the SspI fragments were poorly resolved (see Fig. 2). Summing the AseI or DraI fragments observed with Neo1 DNA yielded genome sizes of $8 \cdot 3$ or $8.6 \mathrm{Mb}$, respectively. For Neo2, size estimates with the two enzymes were 8.3 and $9.9 \mathrm{Mb}$, respectively. Similar experiments performed with Gamma3 DNA yielded resolved fragments with AseI and DraI (data not shown), from which we could calculate genome sizes of 8.3 and $8.6 \mathrm{Mb}$, respectively. These results indicate that the genome sizes of the Neo and Gamma strains are very similar to those of other filamentous actinomycetes, which range from 8 to $10 \mathrm{Mb}$ (Bentley et al., 2002; Ikeda et al., 2003; Redenbach et al., 2000). 


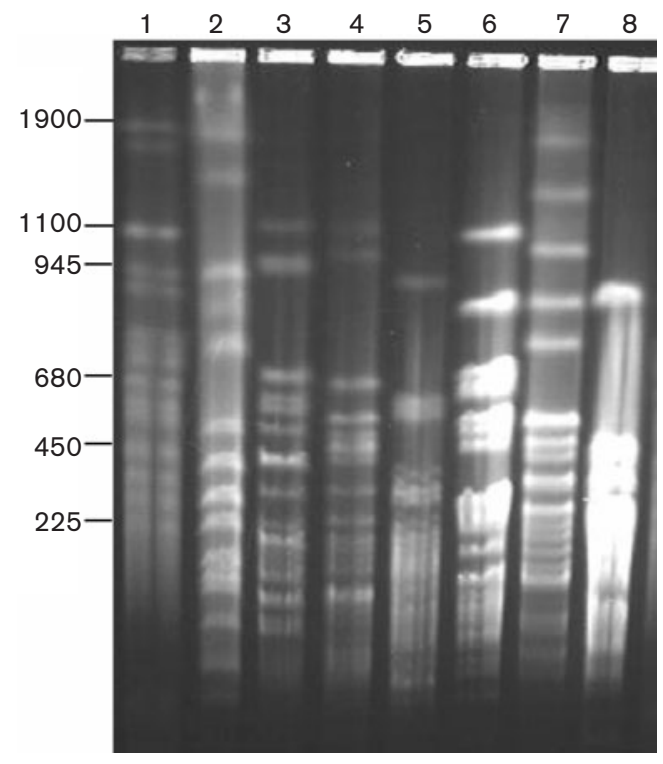

Fig. 2. Pulsed-field gel electrophoresis of $\mathrm{Neo} 1$ and $\mathrm{Neo} 2$ DNA. Lane 1, Saccharomyces cerevisiae chromosomes; lane 2, Asel-digested Streptomyces coelicolor DNA; lanes 3-5, Neo1 DNA digested with Asel (lane 3), Dral (lane 4) or Sspl (lane 5); lanes 6-8, Neo2 DNA, digested with Asel (lane 6), Dral (lane 7) or Sspl (lane 8). Sizes of selected DNA fragments (in kb) are indicated on the left.

\section{Genes for secondary metabolism}

A substantial fraction of the metabolites produced by Streptomyces and related actinomycetes consist of a polyketide or a polypeptide scaffold. Most peptide-derived metabolites are synthesized by NRPSs (Marahiel, 1997), while two major routes exist for polyketide synthesis in actinomycetes (Hopwood, 1997): one employs type I enzymes (PKS-I), also known as modular PKSs; and the other uses type II systems (PKS-II), mostly responsible for the synthesis of aromatic polyketides. Typical of actinomycetes is the presence, in a single strain, of genes encoding PKS-I, PKS-II and NRPSs.

We screened the strains listed in Table 1 for the presence of PKS-I, PKS-II and NRPS genes. All strains analysed yielded a distinct band of the expected size with all primer sets (data not shown). In order to confirm that the amplicons did correspond to the target genes, the products from selected strains were subcloned and sequenced. The results are summarized in Tables 2 and 3. In all cases analysed, the retrieved sequences corresponded to the expected gene segments. The translated DNAs were highly related $(69 \cdot 6-$ $82 \cdot 1 \%$ identity) to known PKS-I or PKS-II sequences, while for NRPSs the identity scores were lower $(35 \cdot 6-68 \cdot 1 \%)$. The lower level of identity of the NRPS segments was expected, since the amplified segment encodes a relatively variable portion of the A domain (Challis et al., 2000; Stachelhaus et al., 1999).

PKS-I and NRPS genes present a modular organization, with a repetition of similar gene segments within a single gene cluster, so each amplified band may consist of different sequences originating from a single cluster. In addition, a single actinomycete strain normally contains more than one PKS-I or NRPS cluster. However, since KS and A domains present considerable sequence variation, primer pairs are seldom universal and can amplify only a fraction of the genes present. Consequently, only a fraction of the entire PKS-I or NRPS modules can be accessed through PCR. Among seven PKS-I clones sequenced (all deriving from strain Neo6), four different sequences were detected (Table 2). For the NRPSs, we analysed 13 independent clones obtained from

Table 2. PKS sequences

\begin{tabular}{|c|c|c|c|c|c|}
\hline \multirow[t]{2}{*}{ Seq. ID ${ }^{\star}$} & \multirow[t]{2}{*}{ Strain } & \multirow[t]{2}{*}{ Accession no. } & \multicolumn{3}{|c|}{ Best match } \\
\hline & & & Accession no. & Organism $\dagger$ & Identity (\%) \\
\hline PKSI-2‡ & Neo6 & AJ865872 & Q07017 & S. antibioticus & $70 \cdot 5$ \\
\hline PKSI-3§ & Neo6 & AJ865873 & Q9S0R4 & S. avermitilis & $76 \cdot 4$ \\
\hline PKSI-8 & Neo6 & AJ865874 & Q846X6 & S. cinnamonensis & $69 \cdot 6$ \\
\hline PKSI-12 & Neo6 & AJ865875 & O52789 & A. mediterranei & $74 \cdot 9$ \\
\hline $\mathrm{KS} \alpha-3 \mathrm{~N} 1$ & Neol & AJ865867 & Q93HM2 & S. avermitilis & $80 \cdot 8$ \\
\hline $\mathrm{KS} \alpha-5 \mathrm{~N} 2 \mathrm{II}$ & $\mathrm{Neo} 2$ & AJ865868 & Q54495 & S. nogalater & $82 \cdot 1$ \\
\hline $\mathrm{KS} \alpha-3 \mathrm{~N} 6$ & Neo6 & AJ865869 & Q02578 & S. curacoi & $79 \cdot 6$ \\
\hline $\mathrm{KS} \alpha-\mathrm{G} 6$ & Gamma6 & AJ865870 & Q02578 & S. curacoi & $78 \cdot 7$ \\
\hline $\mathrm{KS} \alpha-\mathrm{G} 26$ & Gamma26 & AJ865871 & Q9LCH6 & S. venezuelae & $78 \cdot 9$ \\
\hline
\end{tabular}

${ }^{\star} \mathrm{PKSI}$ and $\mathrm{KS} \alpha$ prefixes indicate clones deriving from PKS-I and PKS-II PCRs, respectively.

$\dagger$ Genus names abbreviated as: A., Amycolatopsis; S., Streptomyces.

$¥$ Two other clones have identical sequences.

\$Another clone has an identical sequence.

IIClone 5 from strain $\mathrm{Neo} 3$ has the same sequence. 
Table 3. NRPS sequences from strain Neo6

\begin{tabular}{|c|c|c|c|c|c|}
\hline \multirow[t]{2}{*}{ Seq. ID } & \multirow[t]{2}{*}{ Accession no. } & \multicolumn{3}{|c|}{ Best match } & \multirow[t]{2}{*}{ aa specificity } \\
\hline & & Organism $\dagger$ & Accession no. & Identity (\%) & \\
\hline PS1 & AJ865876 & S. coelicolor & Q9Z4X5 & $54 \cdot 7$ & Asn \\
\hline PS2 & AJ865877 & S. avermitilis & Q93H39 & $68 \cdot 1$ & Ser \\
\hline PS3 & AJ865878 & A. lactamdurans & Q6WS98 & $35 \cdot 6$ & $?$ \\
\hline PS4 & AJ865879 & P. putida & Q88F77 & $42 \cdot 3$ & Asp \\
\hline PS6 & AJ865880 & P. putida & Q88F77 & $43 \cdot 1$ & Asp \\
\hline PS7 & AJ865881 & P. putida & Q88F77 & $44 \cdot 0$ & Asp \\
\hline PS11 & AJ865882 & S. coelicolor & Q9Z4X6 & $51 \cdot 9$ & $?$ \\
\hline PS13 & AJ865883 & P. putida & Q88F77 & $43 \cdot 0$ & Asp \\
\hline PS16 & AJ865884 & P. putida & Q88F78 & $40 \cdot 6$ & Asx \\
\hline
\end{tabular}

${ }^{\star}$ Predicted amino acid activated by the corresponding A domain.

$\dagger$ †enus names abbreviated as: P., Pseudomonas; other abbreviations as in Table 2.

Neo6 DNA, obtaining nine different nucleotide sequences (Table 3). The NRPS primers were designed to amplify most of the DNA segment encoding the amino acid specificity pocket of A domains, including 7 out of 8 diagnostic residues (Challis et al., 2000; Stachelhaus et al., 1999). Thus, sequencing of the amplified products obtained from one strain could provide information on the minimum number of NRPS modules, as well as on the amino acids recognized by the corresponding A domains. The residues lining the binding pocket of the putative Neo6 A domains were determined by alignment with GrsA (CAA33603). Comparison with a database of 442 A domain sequences allowed the identification of the amino acid likely to be recognized by the putative Neo6 A domains (Table 3). Four different domains are likely to activate Asp, and one each is likely to recognize either Asp/Asn, Asn or Ser. Interestingly, for the two remaining domains, the signature sequence had no correspondence in the database, even if both sequences were highly similar to known A domains. Thus, we can conclude that strain Neo6 contains at least nine different A domains, and presumably an equivalent number of NRPS modules, likely to recognize at least three distinct amino acids.

Since PKS-II systems do not have a modular organization, a gene cluster usually encodes a single KS $\alpha$. However, some strains possess more than one PKS-II cluster, and this route is also used for the synthesis of spore pigments (Hopwood, 1997). In a survey of 85 Streptomyces isolates, $60 \%$ could be sequenced directly while the remaining showed multiple sequences that needed cloning (Metsä-Ketelä et al., 2002). To investigate whether a single strain contained more than one PKS-II cluster, some PCR products were directly sequenced. In all instances we observed mixed sequences, suggesting that more than one DNA segment had been amplified. We thus cloned six PCR products and analysed four clones each. All clones deriving from a single strain always yielded the same sequence, which corresponded to the major signal read from direct analysis of the corresponding PCR product (data not shown). We suspect that the analysed strains may encode more than one $\mathrm{KS} \alpha$, but that amplification is biased towards one of them, and several clones should be analysed in order to identify other KS $\alpha$ sequences.

The results from selected PKS-II gene segments are summarized in Table 2. The deduced $\mathrm{KS} \alpha$ sequences from strains Neol and Neo6 were $94 \%$ identical, and those from Gamma6 and Gamma26 were $98 \%$ identical. In addition, two $\mathrm{KS} \alpha$ clones, obtained from strains $\mathrm{Neo} 2$ and $\mathrm{Neo} 3$, had identical sequences. In phylogenetic trees of KS $\alpha$ sequences from various Streptomyces strains, the enzymes for antibiotic biosynthesis were found to clearly separate from those involved in spore pigment formation, although two KS $\alpha$ 's from non-Streptomyces actinomycetes did not belong to any of the two clades (Metsä-Ketelä et al., 2002). When the five different sequences were compared with those of selected $\mathrm{KS} \alpha$ 's, only $\mathrm{KS} \alpha 5-\mathrm{N} 2$ clearly grouped with enzymes for antibiotic biosynthesis (Fig. 3). The other four sequences formed two separate branches in the tree.

\section{Antimicrobial activities}

The production of bioactive metabolites by the strains was assayed by the agar plug method. Strains Neo2, -3 and -6 showed significant activity against B. subtilis, Staph. aureus and M. luteus, while no activity was detected against E. coli. Similarly, Gamma22 produced significant anti-Grampositive activity (data now shown). Occasionally, Neo1 produced a weak activity against E. coli (data not shown).

Chromatographic analysis indicated that $\mathrm{NeO} 2$ and $\mathrm{Neo} 3$ produced different bioactive metabolites (not shown). The active metabolites produced by $\mathrm{Neo} 3$ were extracted with ethyl acetate and analysed, as described in Methods. This led to the identification of an HPLC peak eluting at $24 \cdot 2$ min that contained the major anti-staphylococcal activity. This peak exhibited UV-VIS maxima at 234, 269 and $437 \mathrm{~nm}$, and showed a $[\mathrm{M}-\mathrm{H}]^{-}$signal at $629 \cdot 1$ upon MS analysis. Database searches indicated that these values 


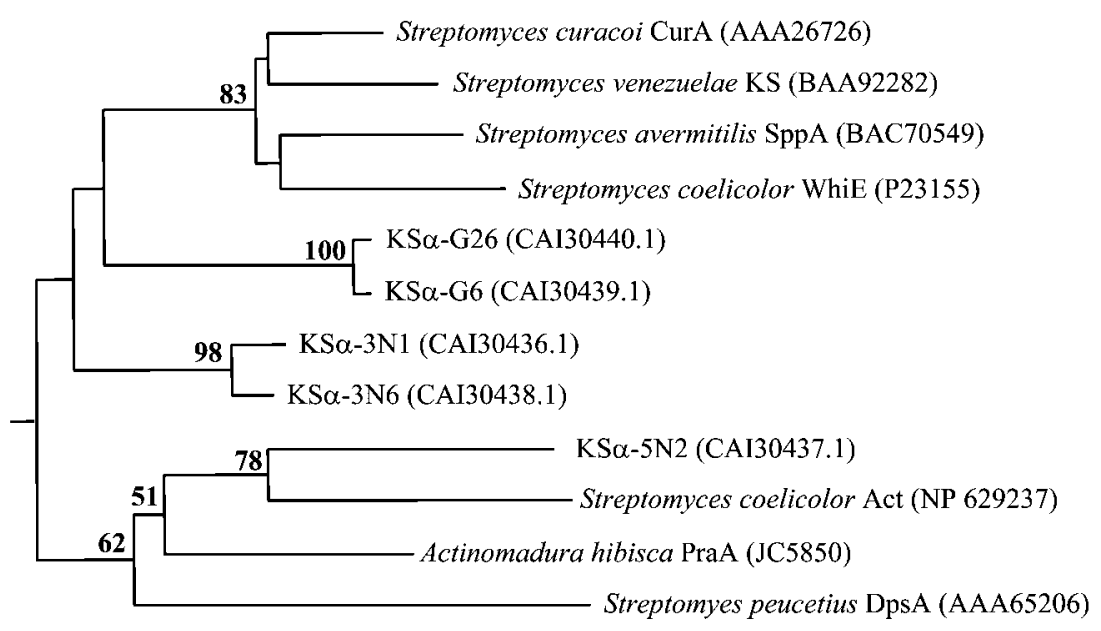

0.1
Fig. 3. Phylogenetic tree of $\mathrm{KS} \alpha$ sequences. The translated sequences of the $\mathrm{KS} \alpha$ clones (Table 2) were aligned over a 145 aa span with selected actinomycete $\mathrm{KS} \alpha$ sequences. Bootstrap values greater than $50 \%$ are shown at nodes. The tree was rooted using the sequence of a type I PKS (Q07017, KS from module 5 of the Streptomyces antibioticus oleandomycin PKS) as outgroup. The scale bar indicates 10 inferred substitutions per 100 aa. CurA, WhiE and SppA are responsible for spore pigment formation, while Act, PraA and DpsA are involved in the synthesis of actinorhodin, pradimicin and daunorubicin, respectively. Accession numbers of reference sequences are indicated. closely matched those of the bioactive dimeric isochromanequinone GTRI-BB, which has a molecular mass of 630, a UV-VIS spectrum compatible with that observed for the $24 \cdot 2$ min peak, and is reported to have anti-Gram-positive but not anti-Gram-negative activity (Yeo et al., 2002). Thus, it is quite likely that Neo3 produces this same compound. Using the same methodology, the active peak produced by Gamma22 did not find a match with any database entry, indicating that it represents a potentially new antibiotic.

\section{Distribution of Neo strains in different soils}

The Neo-specific PCR protocol described in Methods was employed to screen soil DNAs for the presence of 16S rRNA sequences ascribable to Neo strains. We selected several soil samples from different environments, and of different $\mathrm{pH}$. In total, 100 soil DNAs were surveyed by PCR analysis. We could detect a distinct signal from $33 \%$ of the soils with a $\mathrm{pH}$ of 5.5 or lower. No signal was observed from 19 soils with $\mathrm{pH}>7$. From this analysis, the Neo strains that can be targeted by our PCR conditions appear to be present above the detection limit only in acidic soils. This result is consistent with their growth behaviour as acidophiles (Busti et al., 2006). On the other hand, we were able to obtain a PCR product from soil samples collected in distinct locations from different continents (i.e. Europe, Africa and America), indicating that Neo-like sequences are widespread on the planet. This result is consistent with the isolation of the Neorelated Ellin strains from an Australian soil (Joseph et al., 2003).

The 16S rRNA sequences of several strains, identified as 'Acidophilic Actinobacterium', have recently been deposited in databases (e.g. GenBank accession numbers AB180758, $\mathrm{AB} 180762$ and $\mathrm{AB} 180777)$. These sequences are highly related to those of the Gamma strains, suggesting a global distribution for these strains also.

\section{DISCUSSION}

The hypothesis that led us to search for previously uncultured actinomycetes was that these strains were unlikely to have been extensively screened in the past. If they were also able to produce bioactive metabolites, then they should offer a higher probability of discovering novel compounds than previously screened strains (Donadio et al., 2005). While phylogenetic, chemotaxonomic and morphological criteria indicate that Catenulispora and Actinospica strains belong to the order Actinomycetales (Busti et al., 2006; Cavaletti et al., 2006), the ability to produce secondary metabolites is not a uniform trait within this order. In fact, few metabolites have been described as produced by strains belonging to the suborders Actinomycinae, Corynebacterineae, Frankineae, Glycomycineae, Micrococcineae and Propionibacterineae. The data reported here show that at least some strains within the proposed suborder Catenulisporinae share with Streptomyces and related genera several important features, such as the production of antibacterial activities at high frequency, along with the presence within a single strain of genes encoding NRPSs and type I and II PKSs. To our knowledge, the simultaneous presence of these three biosynthetic routes has been reported so far only within the antibioticproducing Actinomycetales. It is also worth pointing out that, within the Actinomycetales, all the prolific producers of secondary metabolites grow as filamentous mycelia, as do the Neo and Gamma strains. In addition, many filamentous actinomycetes possess a chromosome of $8 \mathrm{Mb}$, or higher (Redenbach et al., 2000), as do the Neo and Gamma strains, while mycobacteria and corynebacteria have smaller genomes (Cole et al., 1998; Correia et al., 1994). Thus, there appears to be a correlation between the ability to produce secondary metabolites on the one hand and genome size and filamentous growth on the other.

Recently, Janssen and coworkers (Joseph et al., 2003; Sait et al., 2002) have isolated a large number of previously 
uncultured bacteria. Five of their isolates are highly related to the Neo strains, judging by their $16 \mathrm{~S}$ rRNA gene sequences. Thus, we share the surprise expressed by Sait et al. (2002) that previously uncultured strains could be found also within the intensively isolated actinomycetes. However, the long incubation times required for the appearance of Neo and Gamma strains on isolation plates and their acidophilia might have hindered their retrieval during previous isolation campaigns. Recent isolation efforts (Busti et al., 2006; Cavaletti et al., 2006; Donadio et al., 2005; Joseph et al., 2003; Magarvey et al., 2004; Mincer et al., 2002; Sait et al., 2002) indicate that both marine and terrestrial sources can provide hitherto undescribed actinomycete taxa.

Strain Neo3 produces a bioactive molecule with properties indistinguishable from those of the dimeric isochromanequinone GTRI-BB, produced by a Micromonospora sp. (Yeo et al., 2002). It should be noted that compound GTRI-BB is similar to the well-studied Streptomyces coelicolor metabolite actinorhodin, which is synthesized by a PKS-II system (Hopwood, 1997). In addition, Neo3 contains a sequence encoding a KS $\alpha$ related to the actinorhodin enzyme (Fig. 3). Thus, we infer that $\mathrm{Neo} 3$ contains at least one complete gene cluster for secondary metabolite production. Apparently, GTRI-BB represents one additional example of the same metabolite produced by phylogenetically unrelated strains (Lancini et al., 1995). On the other hand, Gamma22 produces a compound whose mass and UV properties do not find a match in antibiotic databases. Thus, it may represent a novel antibiotic. It should be noted that the fact that Neo3 produces a known bioactive compound does not imply that all secondary metabolites produced by Neo strains will be known compounds, nor is it reasonable to expect that previously uncultured strains would produce novel metabolites only.

In order to provide a reasonable chance of yielding novel antibiotics, strains belonging to novel taxa must: (1) be able to produce secondary metabolites; (2) possess diversified pathways for secondary metabolism; (3) present significant genetic diversity; (4) be retrievable in large numbers; and (5) be amenable to scale-up for large-volume fermentation. We have provided evidence that the Catenulispora and Actinospica strains fulfil criteria 1 and 2, and, from the limited number of isolates analysed, possibly also criterion 3 . Further work is required to establish the true diversity of these new taxa and the ease with which they can be isolated in large numbers. Preliminary data indicate that these strains can be effectively grown in liquid media.

We assume that phylogenetically distant strains are more likely to possess different genes than phylogenetically related strains; since secondary metabolites are produced by the concerted action of several genes, it is reasonable to expect an increased number of novel genes and gene combinations from strains phylogenetically unrelated to strains highly screened for antibiotics. The Neo and Gamma strains described in this work, and other strains currently under investigation in our laboratory (Donadio et al., 2005), represent a potential source of novel antibiotics and other biologically active substances. In our opinion, an expansion of the chemical diversity available for pharmaceutical screening is badly needed, especially in the fight against drug-resistant pathogens. Strains belonging to novel actinomycete taxa might fulfil this role.

\section{ACKNOWLEDGEMENTS}

We are grateful to Julie de Keyser for her contribution to the characterization of the Actinospica strains.

\section{REFERENCES}

Atlas, R. M. \& Park, L. C. (1993). Handbook of Microbiological Media. Boca Raton: CRC Press.

Bentley, S. D., Chater, K. F., Cerdeno-Tarraga, A. M. \& 40 other authors (2002). Complete genome sequence of the model actinomycete Streptomyces coelicolor A3(2). Nature 417, 141-147.

Beyazova, M., Brodsky, B., Shearer, M. \& Horan, A. C. (1995). Preparation of actinomycete DNA for pulse field gel electrophoresis. Int J Syst Bacteriol 45, 852-854.

Bull, A. T., Ward, A. C. \& Goodfellow, M. (2000). Search and discovery strategies for biotechnology: the paradigm shift. Microbiol Mol Biol Rev 64, 573-606.

Busti, E., Cavaletti, L., Monciardini, P., Schumann, P., Rohde, M., Sosio, M. \& Donadio, S. (2006). Catenulispora acidiphila gen. nov., sp. nov., a novel mycelium-forming actinomycete and proposal of Catenulisporaceae fam. nov. Int J Syst Bacteriol (in press)

Cavaletti, L., Monciardini, P., Schumann, P., Rohde, M., Bamonte, R., Busti, E., Sosio, M. \& Donadio, S. (2006). Actinospica acidiphila gen. nov., sp. nov., and Actinospica robiniae gen. nov., sp. nov.; proposal for Actinospicaceae fam. nov. and Catenulisporinae subordo nov. in the order Actinomycetales. Int J Syst Bacteriol (in press)

Challis, G. L., Ravel, J. \& Townsend, C. A. (2000). Predictive, structure-based model of amino acid recognition by nonribosomal peptide synthetase adenylation domains. Chem Biol 7, 211-224.

Cole, S. T., Brosch, R., Parkhill, J. \& 39 other authors (1998). Deciphering the biology of Mycobacterium tuberculosis from the complete genome sequence. Nature 393, 537-544.

Correia, A., Martin, J. F. \& Castro, J. M. (1994). Pulse-field gel electrophoresis of the genome of amino acid producing corynebacteria: chromosome sizes and diversity of restriction pattern. Microbiology 140, 2841-2847.

Courtois, S., Cappellano, C. M., Ball, M. \& 13 other authors (2003). Recombinant environmental libraries provide access to microbial diversity for drug discovery from natural products. Appl Environ Microbiol 69, 49-55.

Donadio, S., Busti, E., Monciardini, P., Bamonte, R., Mazza, P., Sosio, M. \& Cavaletti, L. (2005). Sources of polyketides and nonribosomal peptides. In Biocombinatorial Approaches for Drug Finding, pp. 19-41. Edited by W. Wohlleben, T. Spelling \& B. Müller-Tiemann. Berlin: Ernst Schering Research Foundation, Springer.

Felsenstein, J. (1993). PHYLIP - Phylogeny Inference Package, version 3.5.1. Distributed by the author. University of Washington, Seattle, USA.

Fisher, M. M. \& Triplett, E. W. (1999). Automated approach for ribosomal intergenic spacer analysis of microbial diversity and its 
application to freshwater bacterial communities. Appl Environ Microbiol 65, 4630-4636.

Gerth, K., Pradella, S., Perlova, O., Beyer, S. \& Muller, R. (2003) Myxobacteria: proficient producers of novel natural products with various biological activities - past and future biotechnological aspects with the focus on the genus Sorangium. J Biotechnol 106, 233-253.

Hopwood, D. A. (1997). Genetic contributions to understanding polyketide synthases. Chem Rev 97, 2465-2498.

Ikeda, H., Ishikawa, J., Hanamoto, A., Shinose, M., Kikuchi, H., Shiba, T., Sakaki, Y., Hattori, M. \& Omura, S. (2003). Complete genome sequence and comparative analysis of the industrial microorganism Streptomyces avermitilis. Nat Biotechnol 21, 526-531.

Joseph, S. J., Hugenholtz, P., Sangwan, P., Osborne, C. A. \& Janssen, P. H. (2003). Laboratory cultivation of widespread and previously uncultured soil bacteria. Appl Environ Microbiol 69, 7210-7215.

Kieser, H. M., Kieser, T. \& Hopwood, D. A. (1992). A combined genetic and physical map of the Streptomyces coelicolor A3(2) chromosome. J Bacteriol 174, 5496-5507.

Lancini, G., Parenti, F. \& Gallo, G. (1995). Antibiotics: a Multidisciplinary Approach. New York: Plenum.

Lazzarini, A., Cavaletti, L., Toppo, G. \& Marinelli, F. (2000). Rare genera of actinomycetes as potential producers of new antibiotics. Antonie van Leeuwenhoek 78, 399-405.

Magarvey, N. A., Keller, J. M., Bernan, V., Dworkin, M. \& Sherman, D. H. (2004). Isolation and characterization of novel marine-derived actinomycete taxa rich in bioactive metabolites. Appl Environ Microbiol 70, 7520-7529.

Marahiel, M. (1997). Protein templates for the biosynthesis of peptide antibiotics. Chem Biol 4, 561-567.

Mazza, P., Monciardini, P., Cavaletti, L., Sosio, M. \& Donadio, S. (2003). Diversity of Actinoplanes and related genera isolated from an Italian soil. Microb Ecol 45, 362-372.

Metsä-Ketelä, M., Salo, V., Halo, L., Hautala, A., Hakala, J., Mäntsälä, P. \& Ylihonko, K. (1999). An efficient approach for screening minimal PKS genes from Streptomyces. FEMS Microbiol Lett 180, 1-6.

Metsä-Ketelä, M., Halo, L., Munukka, E., Hakala, J., Mäntsälä, P. \& Ylihonko, K. (2002). Molecular evolution of aromatic polyketides and comparative sequence analysis of polyketide ketosynthase and 16S ribosomal RNA genes from various Streptomyces species. Appl Environ Microbiol 68, 4472-4479.
Mincer, T. J., Jensen, P. R., Kauffman, C. A. \& Fenical, W. (2002). Widespread and persistent populations of a major new marine actinomycete taxon in ocean sediments. Appl Environ Microbiol 68, 5005-5011.

Monciardini, P., Sosio, M., Cavaletti, L., Chiocchini, C. \& Donadio, S. (2002). New PCR primers for the selective amplification of $16 \mathrm{~S}$ rDNA from different groups of actinomycetes. FEMS Microbiol Ecol 42, 419-429.

Omura, S., Ikeda, H., Ishikawa, J. \& 11 other authors (2001). Genome sequence of an industrial microorganism Streptomyces avermitilis: deducing the ability of producing secondary metabolites. Proc Natl Acad Sci U S A 98, 12215-12220.

Parenti, F. \& Coronelli, C. (1979). Members of the genus Actinoplanes and their antibiotics. Annu Rev Microbiol 33, 389-411.

Pearson, W. R. (1990). Rapid and sensitive sequence comparison with FASTP and FASTA. Methods Enzymol 183, 63-98.

Redenbach, M., Sheel, J. \& Schmidt, U. (2000). Chromosome topology and genome size of selected actinomycetes species. Antonie van Leeuwenhoek 78, 227-235.

Sait, M., Hugenholtz, P. \& Janssen, P. H. (2002). Cultivation of globally distributed soil bacteria from phylogenetic lineages previously only detected in cultivation-independent surveys. Environ Microbiol 4, 654-666.

Sambrook, J. \& Russell, D. W. (2001). Molecular Cloning: a Laboratory Manual, 3rd edn. Cold Spring Harbor, NY: Cold Spring Harbor Laboratory.

Sosio, M., Bossi, E., Bianchi, A. \& Donadio, S. (2000). Multiple peptide synthetase gene clusters in actinomycetes. Mol Gen Genet 264, 213-221.

Stachelhaus, T., Mootz, H. D. \& Marahiel, M. A. (1999). The specificity-conferring code of adenylation domains in nonribosomal peptide synthetases. Chem Biol 6, 493-505.

Suzuki, S. I., Okuda, T. \& Komatsubara, S. (2001). Selective isolation and distribution of the genus Planomonospora in soils. Can J Microbiol 47, 253-263.

Wagman, G. H. \& Weinstein, M. J. (1980). Antibiotics from Micromonospora. Annu Rev Microbiol 34, 537-557.

Watve, M. G., Tickoo, R., Jog, M. M. \& Bhole, B. D. (2001). How many antibiotics are produced by the genus Streptomyces? Arch Microbiol 176, 386-390.

Yeo, W. H., Yun, B. S., Kim, Y. S., Yu, S. H., Kim, H. M., Yoo, I. D. \& $\mathrm{Ki}$, Y. H. (2002). GTRI-BB, a new cytotoxic isochromanquinone produced by Micromonospora sp. SA-246. J Antibiot 55, 511-515. 